\title{
Variations in "rescuability" of immunoglobulin molecules from different forms of human lymphoma: implications for anti-idiotype vaccine development
}

\author{
Mercedes Rodríguez-Calvillo, Susana Inogés, Ascensión López-Díaz de Cerio, \\ Natalia Zabalegui, Helena Villanueva, Maurizio Bendandi* \\ Cell Therapy Area, Department of Hematology, University Clinic of Navarre and the Foundation for Applied Medical Research, \\ Avda. Pio XII, 36-31008 Pamplona, Spain
}

Accepted 25 May 2004

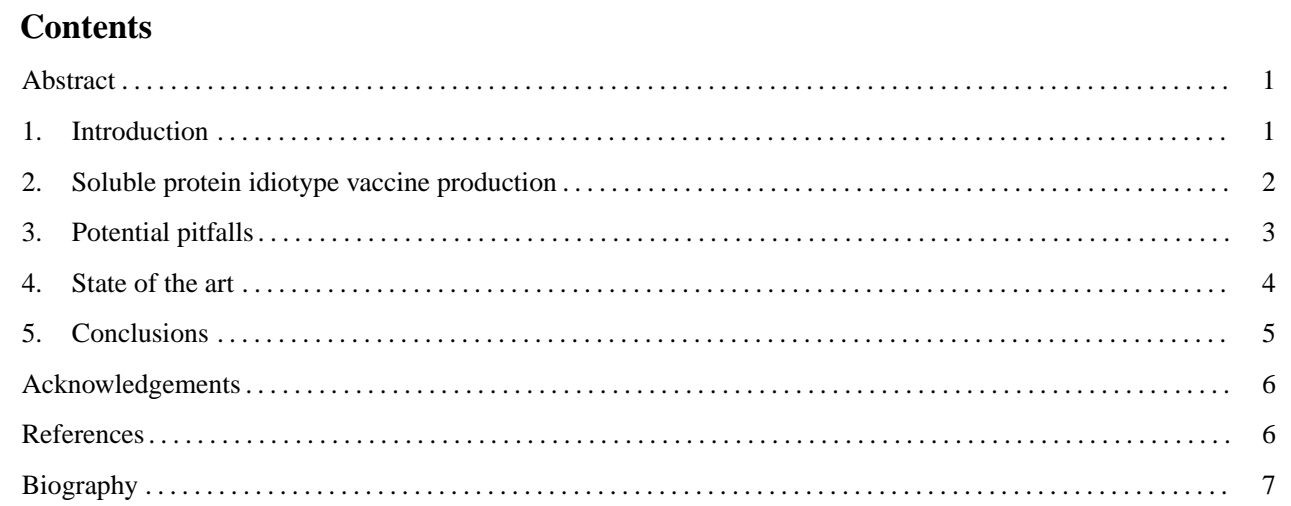

\section{Abstract}

Idiotypic (Id) vaccination has shown promising results in patients with follicular lymphoma (FL). However, it still remains unclear whether the same approach might be suitable for the treatment of other B-cell malignancies. For this reason, we recently performed an interim analysis of patients proposed to receive this treatment at our center.

The feasibility of employing idiotype vaccines was evaluated for five different B-cell malignancies in their first relapse, both in terms of induction and fusion, as well as overall treatment. Our data suggest that, unlike follicular lymphoma (87\%), this approach is not feasible to treat other B-cell malignancies $(0-20 \%)$ such as mantle cell, small lymphocytic, diffuse large cell and Burkitt's lymphoma $(P<0.01)$. The main difficulties encountered were technical problems related to the survival of idiotype-producing hybridomas (83\%) and the early loss of idiotype production by growing hybridomas (17\%).

However, it remains possible that an idiotype vaccine might still be produced through molecular means for most, if not all cases of relapsing B-cell malignancies.

(C) 2004 Elsevier Ireland Ltd. All rights reserved.

Keywords: Idiotype vaccine; Feasibility; B-cell malignancies; Hybridoma; Recombinant technology; bcl-2; PCR

* Corresponding author. Tel.: +34 948 255400x2456; fax: +34948296500.

E-mail address: mbendandi@unav.es (M. Bendandi).

\section{Introduction}

Currently, two ongoing phase-III clinical trials enrolling newly diagnosed patients are evaluating the use of idiotypic (Id) vaccination to treat follicular lymphoma (FL) [1,2]. 
Through these studies, the clinical development of this treatment will approach its final stages should the benefit to patients be demonstrated. However, for this critical step to be reached, it was first necessary to demonstrate that idiotypic determinants present in the immunoglobulin borne by FL cells can serve both as a tumor- and patient-specific antigen (Fig. 1; $[3,4]$ ). Subsequently, it was shown that immunization of FL patients against such a self-antigen was possible [5] and induced an idiotype-specific, humoral [5-8] and cellular $[6,7,9]$ immune response. Finally, it was demonstrated that in vivo, vaccine-induced, Id-specific immune responses can kill FL cells that have survived standard chemotherapy (CHT; $[7,8]$ ).

However, even in the event that one or both of the ongoing clinical trials prove successful, a number of important questions will still remain unanswered. In particular, neither trial will determine whether Id vaccines might be applicable to most if not all relapsed FL and/or other B-cell malignancies. Indeed, the feasibility of using the Id vaccine ultimately depends not only on the capacity to accurately reproduce the very same immunoglobulin ex vivo, including the unique tumor-specific Id, but also on the ability to do so both in a safe manner and in immunotherapeutic quantities.

\section{Soluble protein idiotype vaccine production}

Nowadays, Id vaccines for B-cell malignancies can be divided into two major groups: those based on the whole immunoglobulin as a soluble protein and those based on the DNA sequences encoding both of the immunoglobulin's variable regions (Fig. 1). Here, we shall center on the production of soluble protein, since an extensive discussion of both the methods and the potential of DNA-based Id vaccines falls outside the scope of this review. Nevertheless, it

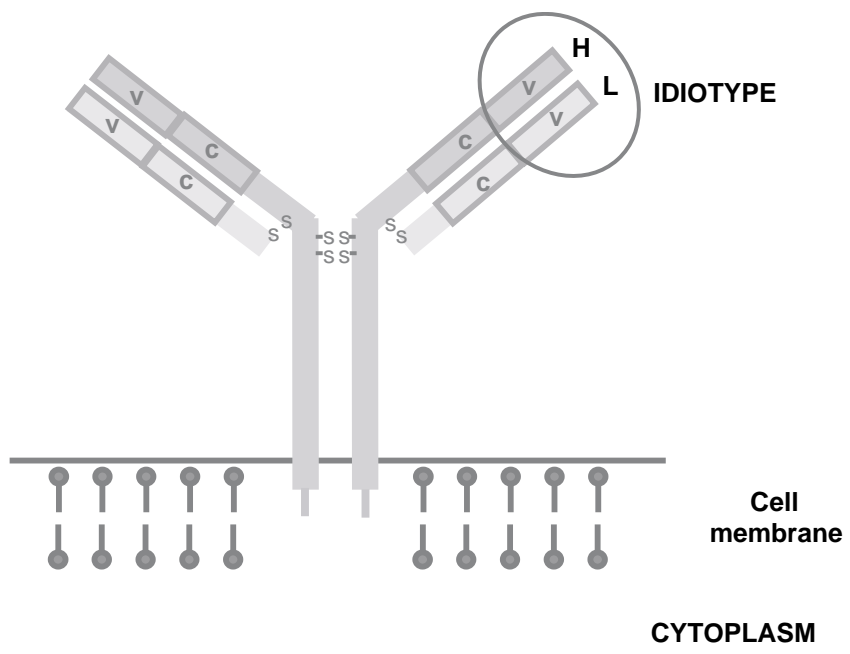

Fig. 1. Schematic representation of idiotype localization within the monomeric immunoglobulin. Abbreviations: V: variable region; C: constant region; H: heavy chain; L: light chain. should be noted that DNA-based Id vaccines [10-14] are at an early stage in their development as clinical tools and deserve to be paid plenty of attention. Indeed, should they ultimately produce results comparable to those of protein-based formulations, they might present a number of potential logistical advantages.

As far as the Id vaccines based on the whole immunoglobulin as a soluble protein are concerned, there are two entirely different methods to obtain them. The more established strategy is based on the use of "rescue" fusions (i.e.: the production of hybridomas), while the newer option is based on recombinant technology. This disregards the option of affinity chromatography purification of a pathologically circulating paraprotein (Fig. 2), which is inapplicable to non-Hodgkin's lymphoma (NHL).

Again, an extensive description of the recombinant technology used to produce the soluble protein falls outside the scope of this review for a number of reasons. Firstly, because all such methods currently under investigation contain a number of steps that remain unpublished due to patent-related issues. Secondly, because our own experience with them is still relatively recent, making it difficult to reach firm conclusions. However, in terms of the fundamental aspects of this approach, a relatively small number of tumor cells can be used to reveal the complete genetic sequence corresponding to both variable regions of the tumor-specific, Id-containing immunoglobulin. Subsequently, these sequences are inserted into especially designed vectors together with non-specific heavy and light chain constant region sequences to express the whole recombinant immunoglobulin in vitro $[1,15]$.

The hybridoma-based methodology used to rescue tumor-specific, Id-containing whole immunoglobulins (Fig. 2) has been responsible for all the Id vaccine-related achievements over the last fifteen years, as outlined above. A large number of tumor cells are required for the fusion with a purposely-selected cell line (K6H6/B5, i.e. ATCC CRL 1823) according to the published methods [16]. Among the several hybridomas grown (Fig. 3), one is ultimately selected according to a number of morphological, genetic, immunological and quantitative features. In particular, the growth of the hybridoma should be relatively fast, and as a single colony to prevent the risk of later discovering that the proliferating cells are not clonal (Fig. 3A). Moreover, the hybridoma molecular fingerprint must fully overlap with that of the corresponding tumor cell to ensure complete identity between the tumor-specific Id and that synthesized by the hybridoma [17]. Furthermore, the hybridoma must indeed secrete an immunoglobulin as determined by standard ELISA. Finally, sufficient immunoglobulin must be secreted by the hybridoma to guarantee enough material can be obtained for all the vaccine doses as well as the post-vaccine immunological tests.

Whichever the method used, the Id-containing immunoglobulin is subsequently converted into the tumor- and 


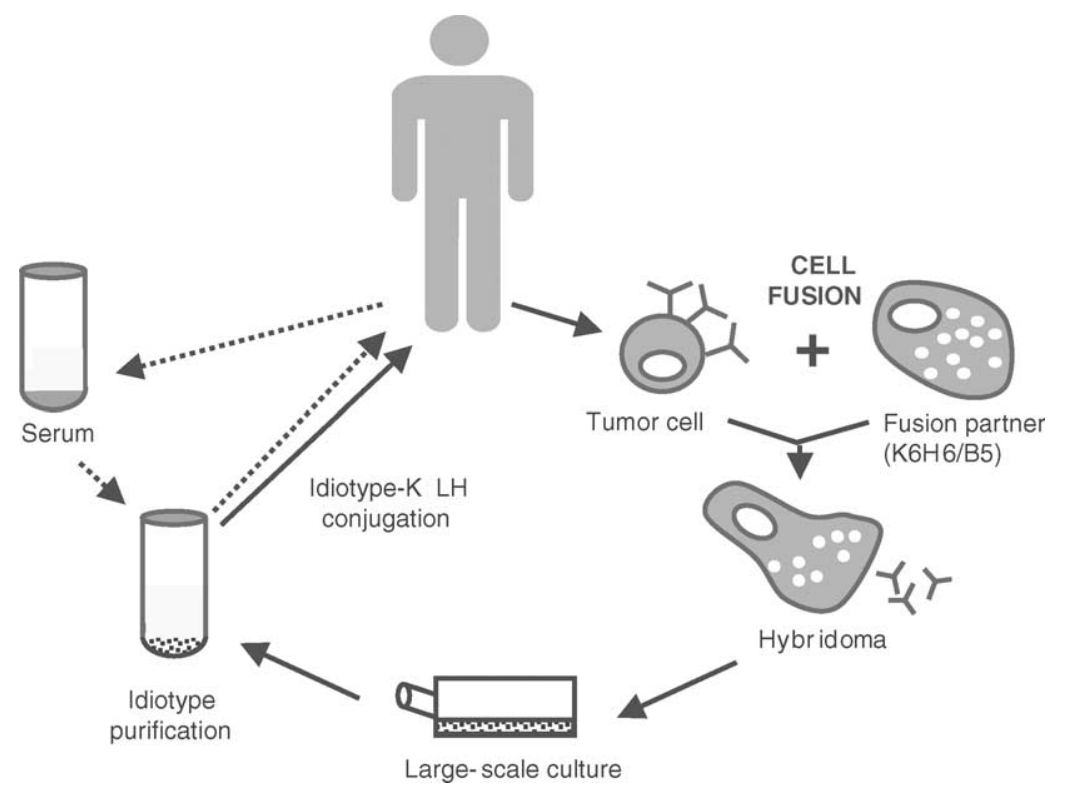

Fig. 2. Flow chart of idiotype vaccine production for B-cell malignancies. The dotted lines refer to the rescue of the idiotype-containing immunoglobulin for B-cell malignancies with a circulating M component. The continuous lines refer to the same rescue carried out by the hybridoma technique for B-cell malignancies with no circulating paraprotein.

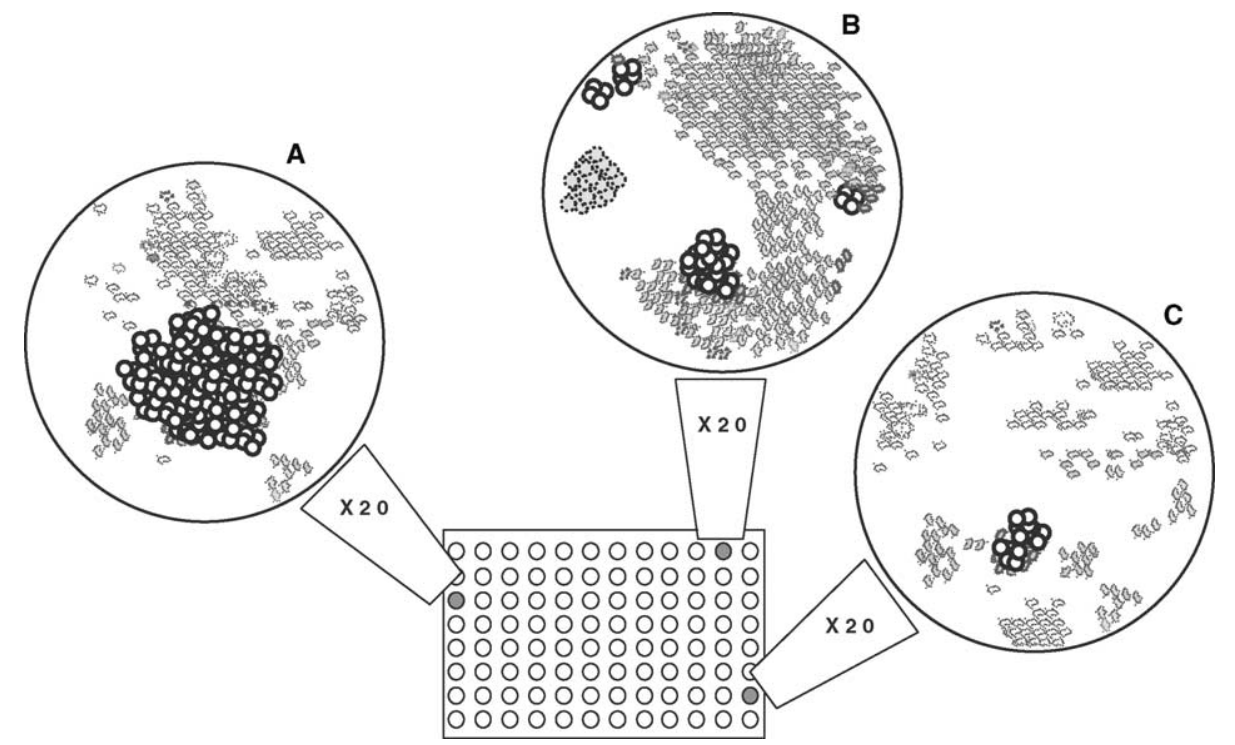

Fig. 3. Schematic representation of the content of individual wells within a 96-well plate 2-3 weeks after a successful hybridoma production. (A) Well containing a clonal, rapidly-growing hybridoma colony. (B) Well containing more than one independent colony of growing hybridomas. (C) Well containing a clonal, slowly-growing hybridoma colony. Of course, wells featuring a combination of these characteristics are also frequently observed.

patient-specific, and therefore custom-made, vaccine through conjugation with a carrier such as KLH and the concomitant administration of an immunological adjuvant such as GM-CSF [2].

\section{Potential pitfalls}

There are still some potential pitfalls that affect both methods currently in use to produce soluble protein Id vaccines.
While some of these pitfalls are common to both techniques, others are particular to one or the other, either being scientific or logistic in nature. However, the most important pitfall is still the feasibility of generating the vaccine in the broadest sense.

In FL, it is reasonable to assume that Id vaccine generation is feasible in about $80 \%$ of cases using hybridoma technology and in about $95 \%$ of cases using recombinant technology. In other NHLs, these figures remain to be determined, although some insights into this problem will be presented 
below. The successful production of an Id vaccine may well take up to six months when based on rescue fusions, as opposed to the three months required when recombinant technology is used [1]. Moreover, rescue fusions require more tumor cells, which often translates into the need for more invasive surgery.

On the other hand, the production of rescue fusions is now a fairly standard and well-optimized technique. It is both fully reproducible and reliable, in contrast to recombinant technology that is still characterized, for better or for worse, by a constant and secret quest for faster, easier and less expensive means of production.

Finally, both strategies face a daily struggle to progress within an intrinsically hostile environment (at least in terms of funding), as custom-made therapeutic solutions are by their very nature difficult for most traditional pharmaceutical companies to exploit. As a consequence, there are very few centers around the world that are willing to dedicate the necessary resources, both in terms of GMP facilities and specialized personnel, to this particular field of clinical investigation.

\section{State of the art}

Until very recently, nothing was known about the feasibility of using idiotypic vaccination for NHLs other than FL. However, last year data from an interim analysis surfaced in the scientific literature, questioning this very possibility even before focusing on either the immunological or clinical efficacy. First of all, our group reported the feasibility of using specific idiotypic vaccination for five different NHL subtypes [18]. All the local IRB-approved, Id vaccine clinical trials ongoing at our institution at that time were evaluated based both on a single, principal endpoint, that is the ability to administer an Id vaccine according to an intention-to-treat. Inclusion was based on an attempt to produce an Id vaccine only at the time of first relapse and on the previous, formal demonstration of the presence of a complete, clonal and tumor-specific immunoglobulin on the tumor cell surface. The diagnosis at relapse was always confirmed pathologically and Id rescue was always carried out according to the traditional hybridoma technique laid out above [16].

The Id vaccine treatment was considered unfeasible when the salvage therapy did not induce an adequate response to proceed with Id vaccination (induction treatment-related), when insufficient idiotype was generated to make the vaccine (fusion-related), or overall. All patients received the CHT regime in use at our institution for their respective disease at that time of first relapse. Following their respective protocols, patients with FL, mantle cell lymphoma (MCL) and small lymphocytic lymphoma (SLL) received Id vaccination if they achieved either a complete (CR) or partial (PR) response according to standard clinical criteria. Patients with either diffuse large cell lymphoma (DLCL) or
Burkitt's lymphoma (BL) received Id vaccination only if they achieved a clinical CR. Indeed, given the very different prognostic meaning of $\mathrm{CR}$ and $\mathrm{PR}$ in either indolent or aggressive NHL, Id vaccination was proposed as further treatment for eligible patients with indolent lymphoma and as active immunotherapy-based maintenance treatment for patients with aggressive NHL. Finally, fusion-related feasibility was evaluated by taking into consideration both of the potential causes of failure: short hybridoma survival and loss of Id production.

In total, 34 patients were enrolled in the study and all of them were evaluated on an intention-to-treat basis. Fifteen patients had FL, 10 had indolent lymphoma other than FL and 9 had aggressive NHL. In terms of the feasibility of induction, we found a substantial difference between indolent (80-100\%) and aggressive (40-50\%) NHL subtypes. However, the data were only striking because they referred to very different eligibility criteria for Id vaccination following CHT. In fact, the overall response (OR) to induction treatment for aggressive NHL reached $75-80 \%$ (CR + PR), but in this group only patients achieving $\mathrm{CR}$ were considered eligible to receive Id vaccination.

A far more important restriction on treatment was the production of the Id-secreting hybridoma. Despite plating a comparable number of 96-well plates in all cases, important differences were found between patients with FL and those with any other NHL studied. Specifically, while most FL cases were characterized by a successful fusion at the first attempt, the other NHL cases required as many as five attempts, irrespective of the ultimate outcome of Id production. Similarly, in most FL cases the average number of successful fusions per 96-well plate was well above 15 . In contrast, the number for most if not all the other NHL cases was typically less than five. All the differences between FL cases and those of all other NHL cases, in terms of fusion-related and overall feasibility, as well as those of both indolent and aggressive lymphoma, were statistically significant.

It is also noteworthy that both the fusion-related and the overall feasibility of the Id vaccine treatment for FL in first relapse were comparable with those already described for both newly diagnosed and relapsed patients with the same disease [6,7]. In our series, one FL patient experienced both pre-vaccine disease progression and failure to produce an Id vaccine. Furthermore, no differences in induction treatment-, fusion-related and overall Id vaccine feasibility were observed among the FL cases analyzed with respect to their histological grade [19].

Finally, the main cause for the failure to produce Id vaccine was the poor survival of the hybridoma (15/18 cases), which accounted for $100 \%$ of failures in aggressive lymphoma ( $9 / 9$ cases). Loss of Id secretion by growing hybridomas accounted for only $3 / 18$ cases of failure to produce Id vaccine. Interestingly, the Id vaccine production success rate was substantially lower in cases of MCL, in contrast to what was initially described with the very same 
5'-CNGAGGAGACAGGTTTATGGTGGTTTGACCTTTAGAGAGTTGCTTTACG GGCCTGTTTCAACACAGACCCACCCAGAGCCCTCCTGCCCTCCTTCTCCC CCATACTACTACTACTACGGTATGGACGTCTGGGGCCAAGGGACCCTGGT AACCGTCTCCTCAGG-3'

Fig. 4. Nucleotide sequence of the K6H6/B5-specific bcl-2/IgH rearrangement.

method [16] in newly diagnosed MCL patients. Indeed, researchers at the NCI have recently reported that the use a rescue fusion-related Id vaccine for MCL patients is feasible in $96 \%$ of cases [20]. Since our findings also contrast with those relating to both newly-diagnosed and relapsed FL cases $[6,7,18]$, it is possible that the faster biological evolution of MCL clonal cells may reflect the presence of characteristics that make first relapse MCL cases more closely resemble first-relapse NHL other than FL in terms of the feasibility of producing hybridoma-based Id vaccine. However, it should also be noted that the same concept does not seem to apply to grade-III FL. In fact, at least in our hands, while this FL subset is generally considered to being both more aggressive and more biologically similar to DLCL, in contrast to DLCL no problems were observed in terms of Id vaccine production. Specifically, while the DLCL-related hybridoma fusions failed to survive, all grade-III FL cases (4/4) were characterized by normal hybridoma survival, although in one instance failure was later provoked by the loss of Id production.

In an attempt to explain some of the discrepancies we found regarding the feasibility of producing Id vaccine for the different first-relapse NHLs, we focused our attention on the possible role of the bcl-2/IgH rearrangement within the hybridomas used to rescue the Id-containing immunoglobulin. First of all, we screened all the tumors from patients for the expression of bcl-2 using a published nested polymerase chain reaction-based (PCR) protocol [21]. In the tumor samples, bcl-2 was expressed in 11/15 FL, 2/5 MCL, 2/5 DLCL, 0/5 SLL and 0/5 BL cases. Subsequently, we screened for bcl-2 expression the hybridomas from bcl-2 expressing tumors that had successfully been used to produce the Id vaccine's core. The results were quite straightforward in that the success of the rescue fusion was not dependent on the expression or activity of bcl-2. Indeed, bcl-2 positive hybridomas were found in 7/11 FL, 1/2 MCL and 1/2 DLCL cases, respectively.

Interestingly, all bcl-2 positive hybridomas maintained the K6H6/B5 fusion partner-specific rearrangement in detriment of that derived from the fused tumor cell (as seen by sequencing individual bcl-2; Fig. 4). This seems to imply that the K6H6/B5-specific bcl-2 rearrangement segregates better than that of most NHLs when the two cell types are fused together. However, in relation to this, another anecdotal finding may be of some importance. Independently of any vaccine trial, a 33-year-old woman with bcl-2 positive FL suffered a relapse two years after the first review and confirmed diagnosis. The relapse involved a rare form of aggressive, highly undifferentiated but still bcl-2 positive NHL, secondary DLCL. Since we had frozen single cell suspensions of both the diagnosis and relapse tumor, an attempt was made to produce hybridomas from both samples. As might have been predicted, most FL-derived, long-term-growing and Id-secreting hybridomas were bcl-2 positive and all of these bcl-2 positive hybridomas maintained the $\mathrm{K} 6 \mathrm{H} 6 / \mathrm{B} 5$-derived, bcl-2/IgH rearrangement. $\mathrm{Cu}-$ riously, most undifferentiated NHL-derived, Id-secreting hybridomas that showed long-term growth were also bcl-2 positive. Nevertheless, all of these hybridomas retained the tumor-derived, bcl-2/IgH rearrangement in detriment of that derived from the K6H6/B5 fusion partner. In terms of clinical outcome, the patient died four months after relapse due to the impressively disseminated, high-dose CHT-resistant NHL.

To complete the picture of the feasibility of producing Id vaccines for aggressive NHL, it seems reasonable to underline that investigators exploiting recombinant technology have so far not reported problems in what is the most advanced trial in this area to date [22].

\section{Conclusions}

On the assumption that the ongoing, phase-III clinical trials on idiotypic vaccination for newly-diagnosed FL will successfully demonstrate that Id vaccines produce clinical benefits, it is reasonable to expect an increase in the interest in such immunotherapeutic strategies for both newly-diagnosed and relapsed FL, as well as for other B-cell malignancies. Our data suggest that, in the vast majority of cases, the feasibility of idiotypic vaccination for patients with first-relapse B-cell malignancies strictly depends on the ultimate capacity to produce a viable Id vaccine, rather than on the probability of inducing a clinical response suitable for subsequent idiotypic vaccination. In this respect, first-relapse FL clearly appears more suitable for hybridoma-based Id vaccine production than any other first-relapse NHL subtype tested in our laboratory.

As the same fusion partner (K6H6/B5) was used with all the different malignant B-cell tumor cells [16], these results seem to imply that B-cells other than those constituting FL clonal populations are less prone to originate suitable hybridomas with the same fusion method utilized for FL Id vaccine production. This conclusion is further supported by the fact that only one fusion attempt was required in most FL cases, as opposed to the other NHL subtypes where the pre-established, arbitrary, five-attempt cut-off, was reached in most instances, irrespective of the ultimate Id vaccine production outcome. Furthermore, the number of successful 
fusions with viable hybridomas was also strikingly superior in FL cases when compared to that of any other NHL subtypes. Similarly, the most frequent cause of failure was the short survival of Id-secreting hybridomas, only occasionally observed for FL hybridomas. This phenomenon might imply that the most crucial step in this strategy for producing Id vaccine is the biological stabilization of an Id-secreting hybridoma. This is a problem that, per se, is not easily surmountable when dealing with rescue fusions and might even accelerate the transition towards recombinant technologies $[23,24]$

Finally, at the time of writing it is not clear whether the recent identification of acquired, potential glycosylation sites within the amino acid sequences of tumor-specific immunoglobulins [25-27] may have an impact on either idiotypic vaccination as a whole or the choice of the methods by which the Id-containing immunoglobulin is rescued.

\section{Acknowledgements}

Supported in part by: FIMA (agreement between FIMA and UTE), FIS Ministerio de Salud, RTIC Cáncer C03/10 (Madrid, Spain), Departamento de Educación y Cultura del Gobierno de Navarra (Pamplona, Spain), PIUNA (Pamplona, Spain), Ruzic Research Foundation (Beverly Shores, IN, USA). Maurizio Bendandi is a Leukemia and Lymphoma Society Scholar in Clinical Research.

\section{References}

[1] Timmerman JM. Immunotherapy for lymphomas. Int J Hematol 2003;77:444-55.

[2] Bendandi M. Role of anti-idiotype vaccines in the modern treatment of human follicular lymphoma. Expert Rev Anticancer Ther 2001;1:65-72.

[3] Lynch RG, Graff RJ, Sirisinha S, Simms ES, Eisen HN. Myeloma proteins as tumor-specific transplantation antigens. Proc Natl Acad Sci USA 1972;69:1540-4.

[4] Sugai S, Palmer DW, Talal N, Witz IP. Protective and cellular immune responses to idiotypic determinants on cells from a spontaneous lymphoma of NZB-NZW F1 mice. J Exp Med 1974;140:1547-58.

[5] Kwak LW, Campbell MJ, Czerwinski DK, Hart S, Miller RA, Levy R. Induction of immune responses in patients with B-cell lymphoma against the surface-immunoglobulin idiotype expressed by their tumors. N Engl J Med 1992;327:1209-15.

[6] Hsu FJ, Caspar CB, Czerwinski D, et al. Tumor-specific idiotype vaccines in the treatment of patients with B-cell lymphoma-longterm results of a clinical trial. Blood 1997;89:3129-35.

[7] Bendandi M, Gocke CD, Kobrin CB, et al. Complete molecular remissions induced by patient-specific vaccination plus granulocytemonocyte colony-stimulating factor against lymphoma. Nat Med 1999;5:1171-7.

[8] Barrios Y, Cabrera R, Yanez R, et al. Anti-idiotypic vaccination in the treatment of low-grade B-cell lymphoma. Haematologica 2002;87:400-7.

[9] Nelson EL, Li X, Hsu FJ, et al. Tumor-specific, cytotoxic Tlymphocyte response after idiotype vaccination for B-cell, nonHodgkin's lymphoma. Blood 1996;88:580-9.
[10] Spellerberg MB, Zhu D, Thompsett A, King CA, Hamblin TJ, Stevenson FK. DNA vaccines against lymphoma: promotion of antiidiotypic antibody responses induced by single chain Fv genes by fusion to tetanus toxin fragment C. J Immunol 1997;159:188592.

[11] Hawkins RE, Russell SJ, Marcus R, et al. A pilot study of idiotypic vaccination for follicular B-cell lymphoma using a genetic approach. CRC NO: 92/33. Protocol NO: PH1/027a. Hum Gene Ther 1997;8:1287-99.

[12] King CA, Spellerberg MB, Zhu D, et al. DNA vaccines with singlechain $\mathrm{Fv}$ fused to fragment $\mathrm{C}$ of tetanus toxin induce protective immunity against lymphoma and myeloma. Nat Med 1998;4:12816.

[13] Timmerman JM, Singh G, Hermanson G, et al. Immunogenicity of a plasmid DNA vaccine encoding chimeric idiotype in patients with B-cell lymphoma. Cancer Res 2002;62:5845-52.

[14] McCarthy H, Ottensmeier CH, Hamblin T, Stevenson FK. Antiidiotype vaccines. Br J Haematol 2003;123:770-81.

[15] Timmerman JM, Czerwinski DK, van Beckhoven A. A phase I/II trial to evaluate the immunogenicity of recombinant idiotype protein vaccines for the treatment of non-Hodgkin's lymphoma (NHL). Blood 2000;96:578.

[16] Carroll WL, Thielemans K, Dilley J, Levy R. Mouse $\times$ human heterohybridomas as fusion partners with human B cell tumors. J Immunol Methods 1986;89:61-72.

[17] Bendandi M, Tonelli R, Maffei R, et al. Identification of the B-cell tumor-specific molecular fingerprint using non-radiolabelled PCR consensus primers. Ann Oncol 2001;12:1479-84.

[18] Inoges S, Rodriguez-Calvillo M, Lopez-Diaz de Cerio A, et al. Feasibility of idiotype vaccination in relapsed B-cell malignancies. Haematologica 2003;88:1438-40.

[19] Harris NL, Jaffe ES, Stein H, et al. A revised EuropeanAmerican classification of lymphoid neoplasm: a proposal from the International Lymphoma Study Group. Blood 1994;84:136192.

[20] Wilson WH, Neelapu SS, Rosenwald A, et al. Idiotype vaccine and dose-adjusted EPOCH-Rituximab treatment in untreated mantle cell lymphoma: preliminary report on clinical outcome and analysis of immune response. Blood 2003;102:358a.

[21] Gribben JG, Freedman A, Woo SD, et al. All advanced stage nonHodgkin's lymphomas with a polymerase chain reaction amplifiable breakpoint of bcl-2 have residual cells containing the bcl-2 rearrangement at evaluation and after treatment. Blood 1991;78:327580.

[22] Leonard JP, Vose J, Timmerman JM, et al. Recombinant idiotype$\mathrm{KLH}$ vaccination $\left(\mathrm{MyVax}^{\mathrm{TM}}\right)$ following $\mathrm{CHOP}$ chemotherapy in mantle cell lymphoma. Blood 2003;102:357a.

[23] McCormick AA, Kumagai MH, Hanley K, et al. Rapid production of specific vaccines for lymphoma by expression of the tumor-derived single-chain Fv epitopes in tobacco plants. Proc Natl Acad Sci USA 1999;96:703-8.

[24] Osterroth F, Garbe A, Fisch P, Veelken H. Stimulation of cytotoxic $\mathrm{T}$ cells against idiotype immunoglobulin of malignant lymphoma with protein-pulsed or idiotype-transduced dendritic cells. Blood 2000;95:1342-9.

[25] Zhu D, McCarthy H, Ottensmeier CH, Johnson P, Hamblin TJ, Stevenson FK. Acquisition of potential $N$-glycosylation sites in the immunoglobulin variable region by somatic mutation is a distinctive feature of follicular lymphoma. Blood 2002;99:2562-8.

[26] Zhu D, Ottensmeier CH, Du MQ, McCarthy H, Stevenson FK. Incidence of potential glycosylation sites in immunoglobulin variable regions distinguishes between subsets of Burkitt's lymphoma and mucosa-associated lymphoid tissue lymphoma. Br J Haematol 2003;120:217-22.

[27] Zabalegui N, Lopes-Diaz de Cerio A, Inoqés S, et al. Acquized potential $\mathrm{N}$-glycosylation within the tumor-specific immunoglobin heavy chains of B-cell malignancies. Haematologica 2004;89:541-6. 


\section{Biography}

Maurizio Bendandi, M.D., Ph.D. is currently associate professor of hematology at the University of Navarre Medical School. He is the Head of the Immunotherapy Lab in the Cell Therapy Area at the University Clinic of Navarre, and a Scholar in Clinical Research of the Leukemia and Lymphoma Society. He obtained his MD at the University of Bologna and his Ph.D. at the "Tor Vergata" University of Rome, after spending several years as a Visiting Fellow at the National Cancer Institute of Bethesda. 